Pesq. Vet. Bras. 38(1):15-22, janeiro 2018 DOI: $10.1590 / \mathrm{S} 0100-736 \mathrm{X} 2018000100003$

\title{
Ocorrência de brucelose e tuberculose bovinas no Rio Grande do Sul com base em dados secundários ${ }^{1}$
}

\author{
Bernardo Todeschini ${ }^{2 *}$, Eduardo F. Costa ${ }^{3}$, Waldemir Santiago-Neto ${ }^{3}$, Diego V. Santos ${ }^{3,4}$, \\ Ana Cláudia M. Groff ${ }^{5}$, Mauro R. Borba ${ }^{3}$ e Luís G. Corbellini ${ }^{3}$
}

\begin{abstract}
Todeschini B., Costa E.F., Santiago-Neto W., Santos D.V., Goff A.C.M., Borba M.R. \& Corbellini L.G. 2018. [Occurrence of bovine brucellosis and tuberculosis in Rio Grande do Sul, Brazil, based on secondary data.] Ocorrência de brucelose e tuberculose bovinas no Rio Grande do Sul com base em dados secundários. Pesquisa Veterinária Brasileira 38(1):15-22. Serviço de Saúde Animal, Superintendência Federal do Rio Grande do Sul, Ministério da Agricultura, Pecuária e Abastecimento, Avenida Loureiro da Silva 515/506, Porto Alegre, RS 90010-420, Brazil. E-mail: bernardo.todeschini@agricultura.gov.br

Results of tests for the diagnosis of bovine brucellosis and tuberculosis were evaluated aiming to analyze the distribution of positivity and characteristics of performance of the control program. The analysis used as secondary data the results of all diagnostic tests of bovines executed in 2008 in the state of Rio Grande do Sul (RS) by veterinarians authorized to operate under the National Program for Control and Eradication of Animal Brucellosis and Tuberculosis. The data were statistically adjusted to minimize the effect of outliers and missing data. The tests achieved $66.80 \%$ of the 497 municipalities in the RS and included the participation of 165 authorized veterinarians. $40.21 \%$ of tests were carried out in the months of April, September and October, and in $73.90 \%$ of establishments had tests performed for both diseases. In the case of brucellosis it appeared that 35,289 animals were tested, with positivity of $0.25 \%$, while the frequency of positive holdings among the 3,406 tested was $0.94 \%$. Females had higher positivity rate $(0.29 \%$ of 26,724 tested) than males ( $0.13 \%$ of 5,235 tested). Animals between $48-60$ months of age presented higher chance of positivity compared to other age groups (Odds Ratio (OR) $=2.63$; CI 95\% $=1.63-4.26$ ). Dairy cattle represented $62.66 \%$ of tested animals, which were more likely to appear as positive than beef animals (OR $=2.32$; CI $95 \%=1.38-3.90$ ). In addition, $73.74 \%$ of the tested holdings were dairy ones. In the case of tuberculosis, 62,149 animals were tested, distributed in 5,151 holdings, with positivity of $0.87 \%$ and $3.13 \%$, respectively. The chance of positivity increased with age, being that animals older than 48 months presented higher values compared to those found in younger animals (OR $=2.07$; CI 95\% $=1.73-2.48)$. Dairy cattle represented $59.74 \%$ of tested animals, which were more likely to be positive than beef ones (OR $=5.03$; CI 95\% $=4.09-6.94)$. Dairy holdings were $78.50 \%$ of all the tests for tuberculosis. The comparative analysis of this study with previous studies on brucellosis prevalence suggested that current control measures have been effective in reducing the prevalence of this disease in RS. On the other hand, information obtained on tuberculosis can be indicators of the condition of this disease, especially in the lack of prevalence stu-
\end{abstract}

\footnotetext{
${ }^{1}$ Recebido em de 20 de abril de 2016.

Aceito para publicação em 16 de dezembro de 2016.

${ }^{2}$ Serviço de Saúde Animal, Superintendência Federal do Rio Grande do Sul, Ministério da Agricultura, Pecuária e Abastecimento (SFA-RS/MAPA), Avenida Loureiro da Silva 515/506, Centro Histórico, Porto Alegre, RS 90010-420, Brasil. *Autor para correspondência: bernardo.todeschini@ agricultura.gov.br

${ }^{3}$ Laboratório de Epidemiologia Veterinária (EPILAB), Departamento de Medicina Veterinária Preventiva, Universidade Federal do Rio Grande do
}

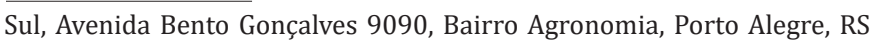
91540-000, Brasil.

${ }^{4}$ Laboratório de Diagnóstico de Doenças dos Animais, Laboratório Nacional Agropecuário, Ministério da Agricultura, Pecuária e Abastecimento (LANAGRO/MAPA), Estrada Ponta Grossa 3036, Porto Alegre, RS 91780580 , Brasil.

${ }^{5}$ Divisão de Fiscalização e Defesa Sanitária Animal, Departamento de Defesa Agropecuária, Secretaria da Agricultura, Pecuária e Irrigação do Estado do Rio Grande do Sul, Avenida Getúlio Vargas 1384, Menino Deus, Porto Alegre, RS 90150-004,Brasil. 
dies carried out in the last 30 years. Finally, it is concluded that the use of secondary data, if properly adjusted, can be an effective tool in the management of animal health programs and monitoring and surveillance systems.

INDEX TERMS: Brucellosis, tuberculosis, PNCEBT, secondary data, monitoring and surveillance systems, animal health, bacterioses.

RESUMO.- Avaliaram-se os resultados de testes para diagnóstico da brucelose e tuberculose bovinas objetivando analisar a distribuição de positividade e características de performance do programa de controle. A análise utilizou como dados secundários todos os resultados de testes para diagnóstico realizados em bovinos no ano de 2008 no estado do Rio Grande do Sul (RS) por médicos veterinários habilitados para atuação no âmbito do Programa Nacional de Controle e Erradicação de Brucelose e Tuberculose Animal. Os dados foram estatisticamente ajustados para minimizar os efeitos de valores extremos e de dados faltantes. Os testes alcançaram $66,80 \%$ dos 497 municípios do RS e incluíram a participação de 165 médicos veterinários habilitados. $40,21 \%$ dos testes foram realizados nos meses de abril, setembro e outubro, sendo que em $73,90 \%$ dos estabelecimentos foram realizados testes para ambas as enfermidades. No caso da brucelose verificou-se que foram testados 35.289 animais, com uma frequência de positivos de $0,25 \%$, enquanto a frequência de estabelecimentos positivos entre os 3.406 testados foi $0,94 \%$. Fêmeas apresentaram frequência de positividade mais elevada $(0,29 \%$, de 26.724 testadas) do que machos $(0,13 \%$, de 5.235 testados). Animais entre 48-60 meses de idade apresentaram chance de positividade superior às demais faixas etárias (Razão de Chances $(\mathrm{RC})=2,63$; IC 95\%=1,63-4,26). Animais de aptidão leiteira representaram $62,66 \%$ dos animais testados, e apresentaram maior chance de positividade do que animais de corte (RC=2,32; IC 95\%=1,38-3,90). Adicionalmente, $73,74 \%$ dos estabelecimentos testados eram dedicados à produção de leite. Já no caso da tuberculose foram testados 62.149 animais distribuídos em 5.151 propriedades, com frequência de positivos de $0,87 \%$ e $3,13 \%$, respectivamente. A chance de positividade aumentou com a idade, sendo que animais com idade acima de 48 meses de idades apresentaram valores superiores aqueles verificados em animais mais jovens (RC=2,07; IC 95\%=1,73-2,48). 59,74\% dos animais testados eram de aptidão leiteira, os quais apresentaram mais chance de serem positivos do que aqueles de corte $(\mathrm{RC}=5,03$; IC 95\%=4,09-6,94). Propriedades leiteiras representaram $78,50 \%$ da totalidade dos testes para tuberculose. A análise comparativa do presente trabalho com estudos precedentes de prevalência da brucelose sugere que as ações de controle em curso têm sido efetivas na redução da prevalência da enfermidade no RS. Por outro lado, as informações obtidas no âmbito da tuberculose podem ser indicadoras da condição desta enfermidade, especialmente pela inexistência de estudos de prevalência realizados há menos de 30 anos. Adicionalmente, conclui-se que a utilização de dados secundários, desde que devidamente ajustados, pode ser uma ferramenta eficaz na gestão de programas de saúde animal e em sistemas de monitoramento e vigilância.

TERMOS DE INDEXAÇÃO: Brucelose, tuberculose, PNCEBT, dados secundários, sistemas de monitoria e vigilância, saúde animal, bacterioses.

\section{INTRODUÇÃO}

A brucelose e a tuberculose bovinas são enfermidades zoonóticas globalmente distribuídas, causadas respectivamente pelas bactérias Brucella abortus (Moreno et al. 2002, WHO 2009) e Mycobacterium bovis (Abrahão 1998, Cosivi et al. 1998). Apesar dos esforços em seu controle, ambas as doenças continuam sendo responsáveis por consideráveis perdas econômicas na pecuária, tanto em nível de produção primária quanto em termos de impactos no comércio de animais e seus produtos (Radostits et al. 2007, Seleem et al. 2010). Além do mais, o controle e erradicação destas enfermidades nas populações animais é considerado decisivo na redução do risco de infecção às populações humanas (Cosivi et al. 1998, Pappas et al. 2006).

Os procedimentos de profilaxia destas enfermidades no Brasil são definidos pelas normativas do Programa Nacional de Controle e Erradicação da Brucelose e da Tuberculose Animal (PNCEBT), instituído em 2001 pelo Ministério da Agricultura, Pecuária e Abastecimento (MAPA) (MAPA 2001). O PNCEBT está estruturado por intermédio de medidas de controle compulsórias complementadas por medidas de adesão voluntária, tais como as normas para certificação de estabelecimentos de status sanitário livre ou monitorado e a habilitação de Médicos Veterinários privados, também denominados Médicos Veterinários Habilitados (MVH), para realização de testes diagnósticos precedidos de capacitação específica e seguindo as definições da Organização Mundial de Saúde Animal (OIE 2009). A legislação nacional determina que os MVH comuniquem mensalmente ao Serviço Veterinário Oficial (SVO) suas atividades relativas ao PNCEBT, mediante remessa de resultados de testes diagnósticos (MAPA 2006). Para brucelose, o teste do Antígeno Acidificado Tamponado (AAT) é a prova de triagem e pode ser realizado por MVH, seguido pelos testes confirmatórios do 2-Mercaptoetanol e Fixação do Complemento, realizados por laboratórios credenciados. Já para tuberculose, os testes de triagem aplicados pelo MVH são o Teste Cervical Simples e o Teste da Prega Caudal, este último podendo ser utilizado em estabelecimentos de pecuária de corte, e a prova confirmatória é o Teste Cervical Comparativo. Estes exames são aplicados em nível animal, podendo estar agrupados em uma bateria de testes, ou seja, vários animais testados de uma mesma propriedade (MAPA 2001).

Os dados informados pelos MVH podem ser enquadrados como parte da coleta passiva de dados, que é um dos componentes dos sistemas de monitoramento e vigilância epidemiológica (SIMVE, tradução do inglês Monitoring and Surveillance Systems) (Salman 2003), com objetivo de geração de informações de interesse para o controle da brucelose e tuberculose bovinas, fornecendo suporte ao processo de tomada decisão quanto às medidas de controle e prevenção (Rushton 2009). Adicionalmente, o SIMVE poderia dar suporte ao alcance de objetivos específicos da vigilân- 
cia epidemiológica, como: rápida detecção de surtos de enfermidades; avaliação de status sanitários de enfermidades ocorrentes ou ausentes; avaliação de condição sanitária de populações determinadas; e avaliação de desempenho do programa de saúde animal em curso (Martin et al. 1987, Thrusfield 2007, Dargatz 2009, Dufour \& Hendrikx 2009, Wells et al. 2009).

Neste contexto, os dados coletados em sistemas de monitoria e vigilância de um programa em saúde animal podem ser considerados como fontes secundárias de dados, os quais são definidos como aqueles gerados com propósitos originalmente distintos daqueles definidos pelo pesquisador para a obtenção da informação desejada (Aaker et al. 1999). A utilização de dados de origem secundária pode resultar em otimização de recursos e eficiência da ação (Salman 2003, Malhotra 2006, Martin et al. 2007), além de incrementar a capilaridade dos sistemas de atenção veterinária (Wells et al. 2009).

Por outro lado, o uso de dados secundários deve considerar suas potenciais restrições, como vieses intrínsecos, qualidade interna e limites inferenciais (Aaker et al. 1999, Malhotra 2006). Neste sentido, o conhecimento, por parte do pesquisador, de aspectos marginais aos dados per se é fundamental para a adequada utilização dos mesmos (Todeschini 2010). A busca de soluções para essas limitações passa pelo conhecimento das bases teóricas do universo de existência e de obtenção dos dados, bem como o adequado tratamento estatístico destes para minimização de efeitos indesejados resultantes do distanciamento existente entre seu objetivo primário e aquele proposto pelo analista (Dohoo et al. 2003, Lattin et al. 2003, Bartlett et al. 2010, Todeschini 2010). Uma limitação frequentemente verificada em séries de dados secundários é a ocorrência de dados faltantes em determinadas variáveis, cuja presença pode representar dificuldades para a análise, como: perda de informações contidas nestes elementos faltantes e complicação no tratamento dos dados (Barnard \& Meng 1999, Schaefer 1999). Não existem determinações rígidas quanto aos tratamentos a serem utilizados, em função dos dados faltantes impactarem no tamanho final da amostra e de suas ocorrências serem multirrelacionais. Dessa forma, a decisão quanto às medidas a serem adotadas passa pelo diagnóstico de níveis de ocorrência e de suas relações com outras variáveis (Todeschini 2010). Neste contexto, a imputação múltipla aparece como alternativa para preenchimento destes dados faltantes com valores plausíveis para a série, e cujo sucesso está relacionado ao adequado estudo do conjunto de dados disponível, objetivando a compreensão dos padrões de ocorrência destas ausências e subsequente definição do método mais adequado para preenchimento das mesmas (Barnard \& Meng 1999, Hair et al. 2006). A definição de variáveis preditoras passa por este processo, bem como a verificação de existência de eventos sistemáticos na ocorrência destas ausências (Todeschini 2010).

Outro ponto de atenção quando da utilização de dados secundários se refere à ocorrência de valores extremos, definidos por Hair et al. (2006) como "observações que apresentam uma combinação única de características identificáveis como distintamente diferentes das demais observações". Os valores extremos não podem ser categoricamente definidos como prejudiciais em um conjunto de dados, embora possam ser bastante influentes em análises estatísticas subsequentes. Seu diagnóstico faz uso de técnicas de detecção bivariadas ou multivariadas, a partir de valores padronizados para as variáveis (Dohoo et al. 2003, Lattin et al. 2003, Hair et al. 2006), sendo fundamental que sejam analisados em seu contexto para compreensão de suas origens e decisão quanto a medidas de mitigação destes efeitos, caso as mesmas sejam necessárias (Lattin et al. 2003).

Os objetivos do presente trabalho foram avaliar a viabilidade de aproveitamento de dados secundários para monitoramento e vigilância da brucelose e da tuberculose bovinas, assim como verificar os padrões de desempenho do PNCEBT no estado do Rio Grande do Sul (RS).

\section{MATERIAL E MÉTODOS}

Foram utilizados dados de todos os testes para diagnóstico de brucelose e tuberculose bovinas realizadas pelos MVH atuantes no âmbito do PNCEBT no RS no ano de 2008, originalmente compilados para desenvolvimento de SIMVE (Corbellini et al. 2010). 0 presente estudo não considerou resultados inconclusivos, somente os resultados finais de cada teste. Os dados obtidos a partir dos documentos originais preenchidos manualmente em atestados cujo modelo encontrava-se previsto pelo Anexo III da Instrução Normativa SDA/MAPA no 30/2006 (MAPA 2006) foram digitados em banco de dados estruturado para esta finalidade a partir do programa Microsoft Access 2007 (Microsoft Corporation 2007). Os dados presentes neste anexo eram: identificação e endereço do proprietário e propriedade, regime de criação e espécie dos animais, data e motivos do teste, dados de referência do antígeno utilizado e dados individuais dos animais testados (identificação individual, sexo, idade, raça, resultado dos testes e destino dado aos animais reagentes). Dados adicionais relacionados à demografia animal e à geografia do RS foram obtidos em repositórios digitais do MAPA, do Instituto Brasileiro de Geografia e Estatística (IBGE 2009) e da Secretaria da Agricultura, Pecuária e Irrigação do estado do Rio Grande do Sul (SEAPI) (SEAPI 2008). Propriedades que realizaram mais de uma bateria de testes naquele ano tiveram somente a primeira delas considerada para fins deste estudo.

Para o diagnóstico de valores extremos de positividade utilizou-se modelo univariado de detecção. Para tanto, o número total de resultados positivos em um mesmo estabelecimento em cada enfermidade foi convertido em escores padronizados $z$ a partir da fórmula (1), cuja distribuição de valores teve hipótese de não-normalidade rejeitada pelos métodos de Kolmogorov-Smirnov e Shapiro-Wilk $(P<0,05)$ (Dohoo et al. 2003, Hair et al. 2006).

$$
z=\frac{x-\mu}{\sigma}
$$

Em que:

$\mathrm{x}=$ número de animais positivos no estabelecimento;

$\mu=$ média de animais positivos no conjunto de estabelecimentos positivos no RS;

$\sigma=$ desvio padrão do número de animais positivos no conjunto de estabelecimentos positivos no RS.

Os valores com escore $z>2,5$ foram considerados como extremos e desencadearam uma busca de informações adicionais nas bases do MAPA e SEAPI sobre suas possíveis causas, as quais registravam focos de brucelose e de tuberculose, assim como as subsequentes ações de saneamento, nas propriedades de ocorrência dos referidos valores extremos. Desta forma, os valores ex- 
tremos foram considerados como derivados de eventos excepcionais, sendo assim substituídos pela média do número de animais positivos no estado $(\mu)$, de acordo com o preconizado por Hair et al. (2006) e Malhotra (2006). Nesta substituição, a seleção dos animais a serem mantidos como positivos, e consequentemente daqueles a serem considerados negativos, foi realizada por intermédio de números aleatórios e dentro das proporções de sexo previamente existentes. Dessa maneira, os quantitativos totais de animais e estabelecimentos testados e de estabelecimentos positivos não foram alterados.

Dados faltantes referentes à idade dos animais foram tratados por imputação múltipla. Para tanto a variável idade foi categorizada em intervalos de 12 meses. 0 modelo utilizou como variáveis preditoras: aptidão, mês do teste, sexo, motivo do teste, cujos níveis de preenchimento em suas próprias séries eram respectivamente $96,81 \%, 100 \%, 89,04 \%$ e $61,82 \%$. Foi utilizado o método de Markov Chain Monte Carlo, sendo as variáveis preditoras inseridas no modelo em uma ordem decrescente de nível de preenchimento em sua própria série (Dargatz \& Hill 1996, Hair et al. 2006, Nunes 2007). 0 conjunto de variáveis foi submetido a 1.000 iterações de regressão logística e regressão linear múltipla.

A força de associação entre as variáveis sexo, aptidão e idade e a ocorrência de brucelose e de tuberculose bovinas foi medida através do cálculo da Razão de Chances (RC), com nível de significância de 5\%. Estas variáveis foram selecionadas em função de sua citação na literatura de referência como medidas potencialmente associadas à ocorrência destas enfermidades (Humblet et al. 2009, Farouk et al. 2011, Matope et al. 2011, Skuce et al. 2012, Brooks-Pollock et al. 2013, Anka et al. 2014).

As análises estatísticas foram realizadas utilizando os programas Microsoft Excel 2007 (Microsoft Corporation 2007), IBM SPSS Statistics 20.0 (IBM Inc. 2011) e Epitools (AusVet Animal Health Services \& Australian Biosecurity CRC 2014). A análise espacial descritiva foi realizada no programa QGIS 2.6 (QGIS 2014).

\section{RESULTADOS}

No ano de 2008 os testes realizados no âmbito do PNCEBT alcançaram 332 municípios do RS e contaram com a participação de 165 MVH. Em 73,90\% dos estabelecimentos foram realizados testes para ambas as enfermidades compreendidas pelo Programa, dos quais $40,21 \%$ foram realizados nos meses de abril, setembro e outubro. 0 motivo predominante informado para realização dos testes foi comercialização $(70,80 \%)$, seguidos de processos de certificação previstos pelo PNCEBT $(17,62 \%)$ e interesse sanitário $(11,54 \%)$.

No caso da brucelose, foram testados 35.289 animais, com frequência de positividade de $0,25 \%$. Fêmeas representaram $74,45 \%$ dos testes e apresentaram frequência de positividade mais elevada $(0,29 \%)$ do que machos $(0,13 \%)$ $(\mathrm{RC}=2,18$; IC 95\%=1,80 - 4,00). Em termos de idade, verificou-se que $50,36 \%$ dos testes foram realizados em animais entre 12-36 meses. Fêmeas apresentaram frequências de teste relativamente uniformes ao longo das idades, ao passo que $86,6 \%$ dos machos foram testados até 36 meses de idade. A chance de positividade de animais entre 48-60 meses de idade foi superior às demais faixas etárias $(\mathrm{RC}=2,63$; IC $95 \%=1,63-4,26$ ). Observou-se que $62,66 \%$ dos animais testados eram de aptidão leiteira, os quais apresentaram diferença significativa de soropositividade quando comparados à aptidão corte $(\mathrm{RC}=2,32$; IC $95 \%=1,38$ $-3,90)$. Em termos de propriedade, foram testados 3.406 estabelecimentos, com frequência de positividade (i.e., pelo menos um animal soropositivo) de $0,94 \%$. Adicionalmente, $73,74 \%$ dos estabelecimentos testados eram dedicados à produção de leite.

No que se refere à tuberculose, foram testados 62.149 animais em 5.151 propriedades, com frequência de positivos de $0,87 \%$ e $3,13 \%$, respectivamente. Fêmeas representaram $83,98 \%$ da totalidade dos animais testados, e similarmente à brucelose, apresentaram chances superiores de positividade do que os machos ( $\mathrm{RC}=2,22$; IC $95 \%=1,62$ $3,03)$. No que se refere à idade, foi verificado que $64,32 \%$ das fêmeas e $87,28 \%$ dos machos testados tinham até 36 meses de idade, sendo que animais com mais de 48 meses de idade apresentaram chance de positividade superior àquela verificada em animais mais jovens $(\mathrm{RC}=2,07$; IC $95 \%=1,73-2,48)$. Animais de aptidão leiteira representaram $59,74 \%$ dos animais testados, os quais apresentaram mais chance de positividade do que aqueles de corte (RC=5,03; IC 95\%=4,09-6,94). Propriedades leiteiras representaram $78,50 \%$ da totalidade dos testes.

No que se refere às mesorregiões, verificou-se que o percentual de municípios testados dentro de cada uma delas variou de $64,80 \%$ a $84,20 \%$. No caso da brucelose, verificou-se maiores frequências de positividade em animais nas mesorregiões Nordeste $(0,71 \%)$ e Metropolitana (0,63\%) (Fig.1).

No caso de estabelecimentos, frequências mais elevadas foram encontradas nas mesorregiões Metropolitana e Centro-Ocidental $(2,55 \%$ e $1,74 \%$, respectivamente), tendo as demais apresentado frequências inferiores a 1,03\% (Fig.2).

Para a tuberculose, verificou-se maiores frequências de positividade em animais nas mesorregiões Nordeste e Centro-Oriental (1,73\% e 1,56\%, respectivamente) (Fig.3), enquanto em estabelecimentos as frequências mais elevadas foram observadas nas mesorregiões Centro-Oriental (7,97\%) e Metropolitana (7,16\%) (Fig.4).

0 diagnóstico de valores extremos, referente ao percentual de animais positivos em um mesmo estabelecimento, localizou dois valores para brucelose e um para tuberculose, os quais foram substituídos por valores médios da série e assim integrados aos resultados.

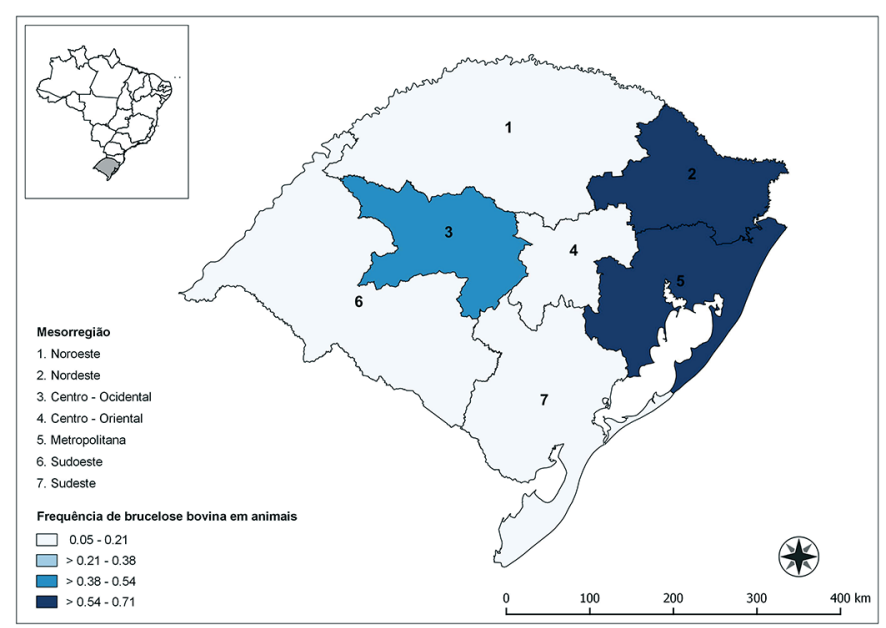

Fig.1. Percentual de positividade para brucelose em animais por mesorregião. 


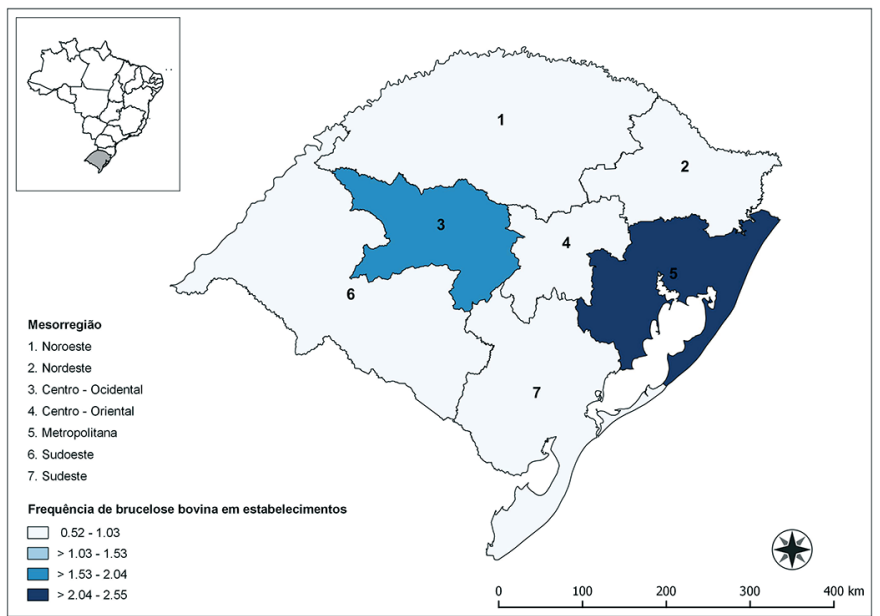

Fig.2. Percentual de positividade para brucelose em estabelecimentos por mesorregião.

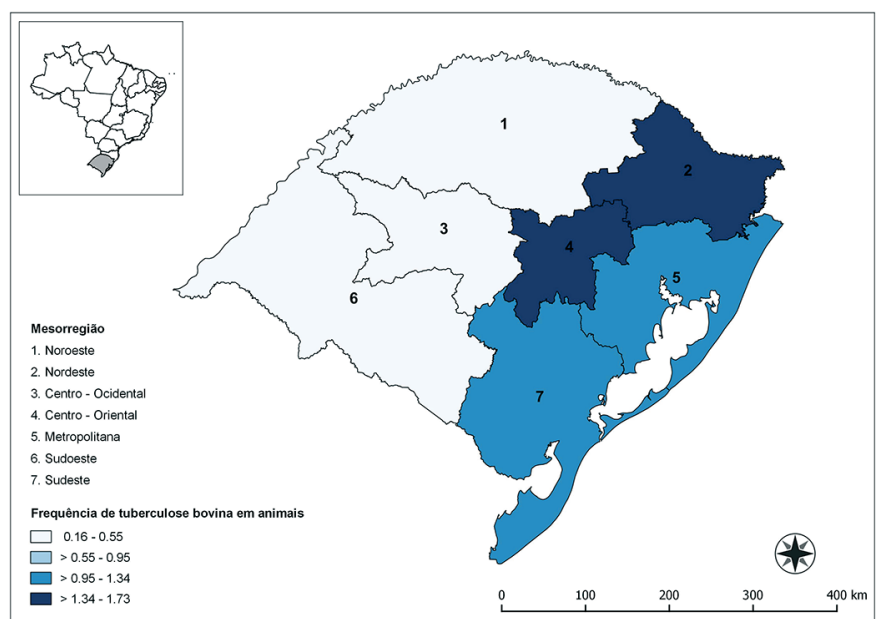

Fig.3. Percentual de positividade para tuberculose em animais por mesorregião.

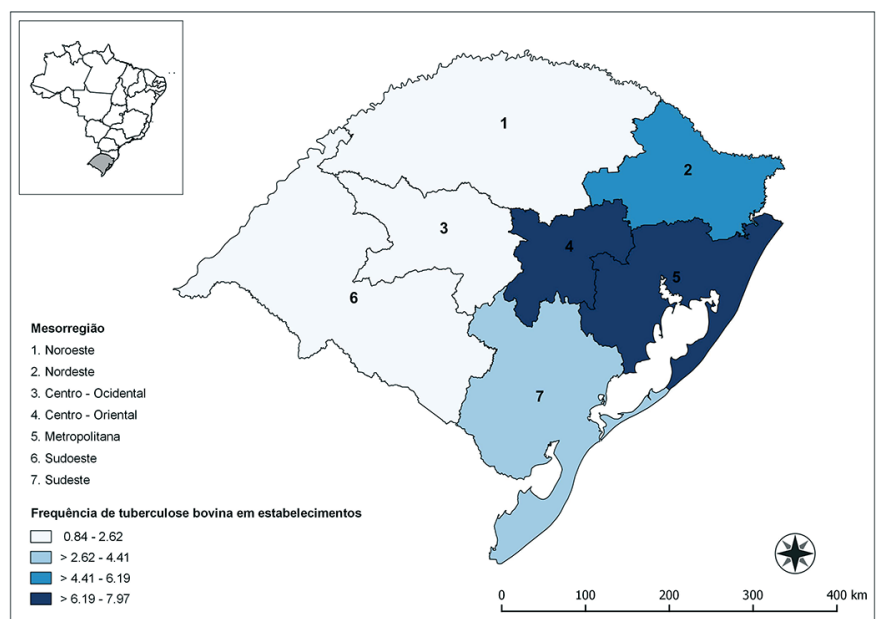

Fig.4. Percentual de positividade para tuberculose em estabelecimentos por mesorregião.

O procedimento de imputação múltipla realizado para a variável idade, que originalmente apresentava $65,20 \%$ de preenchimento, completou os dados faltantes para a mesma, conforme apresentado no Quadro 1.
Quadro 1. Frequências absolutas de preenchimento da variável idade antes e após procedimento de imputação múltipla e percentual de incremento por categoria de idade

\begin{tabular}{cccc}
\hline $\begin{array}{c}\text { Categoria } \\
\text { (meses) }\end{array}$ & $\mathrm{FA}^{\text {a }}$ pré imputação & $\mathrm{FA}^{\mathrm{a}}$ pós imputação & $\begin{array}{c}\text { Incremento } \\
(\%)\end{array}$ \\
\hline$<12$ & 10.865 & 14.344 & 32,0 \\
$12-24$ & 12.762 & 16.220 & 27,1 \\
$24-36$ & 8.672 & 12.570 & 44,9 \\
$36-48$ & 4.955 & 8.140 & 64,3 \\
$48-60$ & 4.015 & 6.007 & 49,6 \\
$60-72$ & 2.298 & 3.186 & 38,6 \\
$>72$ & 2.448 & 2.759 & 12,7 \\
Total & 41.269 & 63.226 & 53,2
\end{tabular}

${ }^{a}$ Frequência absoluta.

\section{DISCUSSÃo}

0 adequado enfoque aos dados originários de vigilância através de dados coletados de forma passiva pode permitir a geração de informações epidemiológicas relevantes. Sua utilização parte da percepção de que há um universo limitado de recursos e tempo, sendo necessária sinergia de esforços e otimização de custos entre os distintos níveis de atuação em sistemas de monitoria e vigilância epidemiológica - SIMVE (Christensen \& Gardner 2000, Murray 2004, Wells et al. 2009). Procedimentos tradicionais de inquérito de prevalência podem apresentar dificuldades operacionais e custos frequentemente elevados, tornando raros os estudos sistemáticos no Brasil (Poester et al. 2009). Neste contexto, a questão passa essencialmente por desenvolver uma metodologia de análise e interpretação adequada para os dados oriundos de coleta passiva dos dados (Fischer et al. 2001, Perez et al. 2002, Ebel et al. 2008, Animal Health Australia 2010).

Dessa forma, o presente trabalho buscou analisar de uma maneira adequada a série de dados disponível. No caso de valores extremos, foram identificados dados cuja distribuição divergia da normalidade esperada para valores da amostra, os quais poderiam gerar vieses inferenciais ao introduzir informações de ocorrências de exceção à série. Dados de estabelecimentos em que a prevalência intra-rebanho seja muito superior à média, como em surtos epidêmicos ou doença endêmica circulante há muito tempo na propriedade, tratados através de frequência simples ao longo do tempo podem falsamente incriminar um aumento da ocorrência do indicador em nível animal. A metodologia utilizada trouxe os dados à normalidade necessária para que as frequências observadas pudessem ser aproximadas da real expressão da enfermidade na população. Foi ainda observado que a padronização dos valores da série em escores $z$, que fez os mesmos passarem a ser expressos pelo número de desvios padrão que os valores originais se situam acima ou abaixo da média aritmética, contribuiu para a manipulação dos dados por expressar diretamente a dispersão de uma determinada observação. Cabe destacar que o fato da série se ajustar à distribuição normal possibilitou a utilização do método do escores $z$ (Malhotra 2006, Seo 2006), tendo sido a verificação de normalidade o ponto inicial do processo de diagnóstico e ajuste de valores extremos. Por outro lado, em séries de longo prazo cujos dados de vigilância coletados de forma passiva sejam obtidos longitudinal- 
mente com periodicidade definida, o diagnóstico de valores extremos poderia ser utilizado como mecanismo de alerta, constando em um sistema de vigilância como o limiar para a intervenção do SVO. Em tal situação, os níveis de normalidade do sistema poderiam ser customizados de acordo com níveis de frequência conhecidos, assim como os níveis máximos de tolerância de frequência da enfermidade, de maneira similar àquela utilizada pelos sistemas de vigilância sindrômica (Dórea et al. 2011, Perrin et al. 2012). A possibilidade de tal utilização foi verificada no presente estudo, no qual foi constatado que os dois valores extremos que fugiam do padrão esperado ocorreram devido a surtos de brucelose e de tuberculose e suas subsequentes ações de saneamento.

A imputação múltipla da variável idade, similarmente ao tratamento aplicado aos valores extremos, foi realizada para aproximar a série de dados da realidade da população. Tal tratamento apresenta-se como de grande aplicabilidade, considerando que séries de dados secundários frequentemente aparecem com dados faltantes, cuja motivação e padrões de ocorrência podem introduzir vieses na análise, assim como dificultar a realização de inferências importantes para a compreensão do comportamento da ocorrência da enfermidade numa população. A significativa redução no quantitativo de dados faltantes relativos à idade, obtida pelo procedimento empregado, permitiu a extrapolação das conclusões para o nível populacional amostrado. Os critérios empregados na seleção de variáveis preditoras apareceram como ponto determinante na qualidade do resultado, no qual deve ser considerado o nível de ausência de dados faltantes nas mesmas e especialmente sua capacidade de predição da variável resposta, com objetivo de evitar que sejam realizadas inferências com base em confundidores (Dargatz \& Hill 1996, Hair et al. 2006, Nunes 2007). Em termos metodológicos, a utilização de árvores de cenário relacionadas às possíveis interações entre variáveis pode auxiliar o pesquisador na seleção de variáveis capazes de melhor predizer o valor a ser imputado. Além disso, o aumento no quantitativo de variáveis preditoras, preferencialmente relacionadas a distintos blocos de informações, permite que eventuais vieses de preenchimento das mesmas, geradas pelo coletor original dos dados (o MVH, no presente estudo), sejam contornados. Fica claro que idealmente seria mais adequado evitar a ocorrência de dados faltantes, para o que Höhle et al. (2009) recomendam supervisão constante nos sistemas de dados de vigilância coletados de forma passiva, propondo processos que gerem alertas aos gestores para situações nas quais os níveis de dados faltantes ultrapassem limites definidos. Finalmente, é possível concluir que o conhecimento do pesquisador quanto ao universo de existência dos dados e seu contexto de obtenção são fundamentais na bem-sucedida seleção de métodos de ajuste e subsequente decisão quanto à abordagem metodológica a ser empregada.

Quanto aos dados de brucelose bovina no RS no ano de 2008, demonstraram-se frequências de positividade em animais e estabelecimentos de $0,25 \%$ e $0,94 \%$, respectivamente. Pode ser verificada uma situação relativamente uniforme no estado em ambos indicadores, cuja variação por mesorregião foi, respectivamente, de $0,05 \%$ a $0,71 \%$ e de $0,52 \%$ a 2,55\%. 0 último estudo de prevalência para essa enfermidade realizado no RS em 2004, conduzido em estratos com limites geográficos assemelhados aqueles das mesorregiões, encontrou prevalências de animais e de estabelecimentos de 1,02\% e 2,06\%, respectivamente (Marvulo et al. 2009). 0 presente estudo encontrou, de maneira geral, frequências totais e mesorregionais inferiores àquelas verificadas por Marvulo et al. (2009). A implementação compulsória da vacinação nas fêmeas entre 3 e 8 meses a partir de 2005 (MAPA 2004) pode estar relacionada com reduções nas frequências de animais positivos, bem como à redução na amplitude de frequências de positivos entre regiões. A vacinação ainda pode estar relacionada com o fato de animais entre 48 e 60 meses apresentarem maior chance de apresentar resultados positivos, os quais nasceram durante a implantação da vacinação compulsória, e dessa maneira pertencem a uma coorte que pode não ter sido alcançada por adequada cobertura vacinal. Adicionalmente, a implantação do PNCEBT, com aumento no número de animais testados, em especial nas categorias de idade inferiores a 48 meses, pode ter levado ao diagnóstico mais precoce da enfermidade e ao subsequente descarte destes animais, reduzindo as frequências de positivos nas categorias precedentes àquela verificada atualmente como de maior chance. 0 fato de $50,93 \%$ dos testes para brucelose terem sido realizado em animais de 12 a 36 meses de idade transparece preocupação dos MVH e produtores com animais em fase prévia ou inicial de reprodução. Por fim, os níveis de frequência relativos de animais e estabelecimentos positivos verificados sugerem evolução na redução de prevalência de brucelose, sinalizando que no futuro será possível a discussão sobre a transição do programa para a fase de erradicação. As diferenças entre sexo nas frequências relativas de positivos ao longo das distintas categorias de idade podem estar relacionadas com o manejo reprodutivo, considerando que entre fêmeas é possível que o descarte de animais doentes em função de manifestações clínicas como infertilidade ou aborto ocorram sem que os mesmos tenham sido submetidos aos testes. Já a concentração de diagnósticos em machos nas categorias de idade de até 36 meses pode denotar preocupação de técnicos e produtores quando da aquisição e da utilização destes animais para reprodução. 0 fato da brucelose ser uma doença de difícil diagnóstico clínico em machos pode ser a razão pela qual os mesmos apresentam percentuais de positivos mais elevados nos grupos etários entre 36 e 60 meses, precisamente aqueles com menor frequência relativa de animais testados deste sexo.

É necessário salientar a importância da organização e análise contínua de dados coletados acerca dos testes diagnósticos realizados ao longo do tempo a fim de se obter uma série histórica que permita auxiliar na avaliação da evolução do programa de controle de uma doença. A comparação desde tipo de dado de vigilância através do tempo é mais fidedigna, pois se avalia informações obtidas de forma similar. Nesse sentido, a diferença de indicadores de brucelose oriundos de dados secundários com os do inquérito de prevalência pode ser influenciada pela forma que a amostra foi obtida. 
No caso da tuberculose, há carência de estudos para estimação de prevalência da enfermidade em animais e rebanhos no estado, sendo que as principais referências existentes são estudos realizados entre 1970 e 2000, desenvolvidos a partir de amostras de conveniência obtidas em propriedades específicas, dados de inspeção em frigoríficos ou a partir de materiais recebidos para diagnóstico laboratorial em universidades ou instituições de pesquisa. Estes estudos encontraram frequências que variaram entre 0,1 e 3,4\% em animais de corte (Abrahão 1998) e 1,9 e 20,3\% em animais leiteiros (Abrahão et al. 2005), com diferença de frequências entre regiões (Abrahão 1998). Neste contexto de deficiência de informação, o aproveitamento de dados secundários ganha maior importância, pois direciona a tomada de decisão pelos gestores do Programa quanto a potenciais gargalos ou pontos de maior atenção. Assim, destaca-se o fato do presente estudo ter verificado marcada diferença entre regiões, especialmente no que se refere à frequência de propriedades positivas. Ainda é possível constatar existência de maior preocupação com tuberculose do que com brucelose, especificamente no que se refere ao quantitativo de animais e propriedades testadas. Há linearidade na tendência de positividade de animais com o avanço da idade, o que é esperado para uma doença de transmissão horizontal com caráter crônico e longo período de incubação.

Quanto ao motivo de realização do teste, observou-se que $42,62 \%$ dos formulários analisados não apresentava o respectivo campo devidamente preenchido, demonstrando a necessidade de reforço na vigilância de qualidade dos atestados. Ainda assim, o fato de $40,21 \%$ dos animais terem sido testados nos meses abril, setembro e outubro pode indicar que a comercialização representava importante motivação, uma vez que estes meses coincidem com as feiras agropecuárias nas estações de outono e primavera no RS. Esta informação pode ser de grande valia para procedimentos de auditoria do SVO e mesmo para laboratórios produtores de antígenos, objetivando o planejamento de sua produção. Em um contexto mais amplo, há evidente necessidade de maior padronização quanto ao preenchimento de formulários do PNCEBT, em especial no que se refere às informações dependentes de dados do responsável pelos animais, como raça, aptidão e idade. Tal padronização tem reflexo direto na qualidade dos dados, sendo fundamental para o aproveitamento dos mesmos dentro da temporalidade necessária (Salman 2003, Dargatz 2009, Dufour \& Hendrikx 2009). A adoção de estratégias de educação continuada dirigidas aos MVH poderia constituir uma alternativa interessante para sensibilização dos mesmos quanto à importância, para o sistema de atenção veterinária, da integralidade de preenchimento e da fidedignidade de seus relatórios.

Por fim, é possível referir que o conjunto de informações relacionadas ao desempenho do PNCEBT no RS pode ser utilizado pelos gestores do programa para adoção de medidas que possam contribuir para a evolução do mesmo no estado, tanto no que se refere ao controle de qualidade global do processo quanto à identificação de pontos críticos, bem como no diagnóstico de regiões nas quais o pro- grama possa necessitar de intervenção ativa para efetiva implementação (Todeschini et al. 2012).

\section{CONCLUSÕES}

As atividades dos MVH atuantes no PNCEBT em 2008 no RS alcançaram parcela relevante de animais, propriedades rurais e municípios do estado, gerando dados que podem ser utilizados pelo SVO para vigilância epidemiológica da brucelose e tuberculose bovinas e promoção de ações de saúde animal em processos de vigilância direcionada ao risco.

O devido aproveitamento destes dados secundários parte da utilização de técnicas de análise definidas com base no universo de existência dos dados e seus mecanismos de obtenção.

A devida capacitação dos MVH poderia contribuir na melhoria de qualidade dos dados originais, o que se refletiria na qualidade das informações derivadas de análises subsequentes. Adicionalmente, há espaço para a estruturação de sistemas de monitoramento e vigilância que permitam o incremento da capilaridade do sistema de atenção veterinária pela efetiva utilização de dados resultantes de ações de vigilância passiva, com ganhos globais em termos de gestão de processos e em eficiência das ações de profilaxia em saúde animal.

\section{REFERÊNCIAS}

Aaker D.A., Kumar V. \& Day G.S. 1999. Pesquisa de Marketing. Atlas, São Paulo. 745p.

Abrahão R.M.C.M. 1998. Tuberculose humana causada por Mycobacterium bovis: considerações gerais e a importância dos reservatórios animais. Dissertação de Mestrado, Faculdade de Saúde Pública, Universidade de São Paulo, São Paulo, SP. 273p.

Abrahão R.M.C.M., Nogueira P.A. \& Malucelli M.I.C. 2005. 0 comércio clandestino de carne e leite no Brasil e o risco da transmissão da tuberculose bovina e de outras doenças ao homem: um problema de saúde pública. Archs Vet. Sci. 2(10):1-17.

Animal Health Australia 2010. Australian Bovine Tuberculosis Surveillance Project. Disponível em <http:// www.animalhealthaustralia.com. $\mathrm{au} /$ programs/adsp/abtbsp/abtbsp_home.cfm> Acesso em 12 out. 2014.

Anka M.S., Hassan L., Khairani-Bejo S., Zainal M.A., Mohamad R.B., Salleh A. \& Adzhar A. 2014. A case-control study of risk factors for bovine brucellosis seropositivity in Peninsular Malaysia. PLoS ONE 9(9):1-7.

AusVet Animal Health Services \& Australian Biosecurity Cooperative Research Centre for Emerging Infectious Disease 2014. Epitools epidemiological calculators. Acesso em 31 jan. 2016.

Barnard J. \& Meng X.L. 1999. Applications of multiple imputation in medical studies: from AIDS to NHANES. Stat. Methods Med. Res. 8:17-36.

Bartlett P.C., Van Buren J.W., Neterer M. \& Zhou C. 2010. Disease surveillance and referral bias in veterinary medical database. Prev. Vet. Med. 94(3/4):264-271.

Brooks-Pollock E., Conlan A.J.K., Mitchell A.P., Blackwell R., McKinley T.J. \& Wood J.L.N. 2013. Age-dependent patterns of bovine tuberculosis in cattle. Vet. Res. 44:97.

Christensen J. \& Gardner I.A. 2000. Herd-level interpretation of test results for epidemiologic studies of animal diseases. Prev. Vet. Med. 45(1/2):83-106.

Corbellini L.G., Carvalho J.B.P., Nunes L.N., Vasconcellos P.A. \& Todeschini B. 2010. Desenvolvimento de um sistema de monitoria e vigilância para gestão das informações geradas pelo PNCEBT em nível estadual. Anais I Seminário Nacional sobre Brucelose e Tuberculose Animal, Belo Horizonte, $\mathrm{MG}$. 
Cosivi O., Grange J.M., Daborn C.J., Raviglione M.C., FuJikura T., Cousins D., Robinson R.A., Huchzermeyer H.F.A.K., Kantor I. \& Meslin F. 1998. Zoonotic tuberculosis due Mycobacterium bovis in developing countries. Emerg. Infect. Dis. 4(1):59-70.

Dargatz D.A. 2009. What is normal? A field approach to characterizing health and management of the nation's animal populations. Prev. Vet. Med. 88(2):94-100.

Dargatz D.A. \& Hill G.W. 1996. Analysis of survey data. Prev. Vet. Med. 28:225-237.

Dohoo I., Martin W. \& Stryhn H. 2003. Veterinary Epidemiology Research. AVC Inc., Charlottetown. 727p.

Dórea F.C., Sanchez J. \& Revie C.W. 2011. Veterinary syndromic surveillance: current initiatives and potential for development. Prev. Vet. Med. 101(1/2):1-17.

Dufour B. \& Hendrikx P. 2009. Epidemiological Surveillance in Animal Health. 2nd ed. OIE. Paris. 386p.

Ebel E.D., Williams M.S. \& Tomlinson S.M. 2008. Estimating herd prevalence of bovine brucellosis in 46 U.S.A. states using slaughter surveillance. Prev. Vet. Med. 85(3/4):295-316.

Farouk U.M., Salisu I., Ikwe A. \& Bale J.0.O. 2011. Prevalence of bovine brucellosis and risk factors assessment in cattle herds in Jigawa State. ISRN Veterinary Science: 1-4.

Fischer E.A.J., Van Roermund H.J.W., Hemerik L., Van Asseldonk M.A.P.M. \& De Jong M.C.M. 2001. Evaluation of surveillance strategies for bovine tuberculosis (Mycobacterium bovis) using an individual based epidemiological model. Prev. Vet. Med. 67(4):283-301.

Hair J.F., Lack W.C., Babin B.J., Anderson R.E. \& Tatham R.L. 2006. Multivariate Data Analysis. Prentice Hall, New Jersey. 889p.

Höhle M., Paul M. \& Held L. 2009. Statistical approaches to the monitoring and surveillance of infectious diseases for veterinary public health. Prev. Vet. Med. 91(1):2-10.

Humblet M., Boschiroli M.L. \& Saegerman C. 2009. Classification of worldwide bovine tuberculosis risk factors in cattle: a stratified approach. Vet. Res. 40(5):1-24.

IBGE 2009. Perfil dos Municípios Brasileiros. Disponível em <http://www. ibge.gov.br/home/estatistica/economia/perfilmunic/2009/defaulttabzip.shtm> Acesso em 13 out. 2010.

IBM Corp. 2011. IBM SPSS Statistics for Windows, Versao 20.0. IBM Corp., Armonk.

Lattin J.M., Carrol J.D. \& Green P.E. 2003. Analyzing multivariate data. Pacific Grove. Thomson Learning. 555p.

Malhotra N. 2006. Pesquisa de Marketing: uma orientação aplicada. Bookman, Porto Alegre. 720p.

MAPA 2001. Instrução Normativa no 2 de 10 de janeiro de 2001. Disponível em <http://extranet.agricultura.gov.br/sislegis- consulta/ consultarLegislacao.do? operacao=visualizar\&id $=2502>$ Acesso em 13 out. 2014.

MAPA 2004. Instrução Normativa no 6 de 8 de janeiro de 2004. Disponível em <http://sistemasweb.agricultura.gov.br/sislegis/action/detalhaAto.do?method=consultarLegislacaoFederal $>$ Acesso em 13 out. 2014.

MAPA 2006. Instrução Normativa no 30 de 07 de junho de 2006. Disponível em <http://extranet. agricultura.gov.br/sislegis-consulta/consultarLegislacao.do?operacao=visualizar\&id=16961> Acesso em 13 out. 2014.

Martin P.A.J., Cameron A.R., Barfod K., Sergeant E.S.G. \& Greiner M. 2007. Demonstrating freedom from disease using multiple complex data sources. 2. Classical swine fever in Denmark: case study. Prev. Vet. Med. 79(2/4):98-115.

Martin S.W., Meek A.H. \& Willeberg P. 1987. Veterinary Epidemiology. Iowa State University Press, Iowa. 343p.

Marvulo M.F.V., Ferreira F., Dias R.A., Amaku M., Groff A.C.M., Gonçalves V.S.P., Figueiredo V.C.F., Lôbo J.R. \& Ferreira Neto J.S. 2009. Situação epidemiológica da brucelose bovina no Estado do Rio Grande do Sul. Arq. Bras. Med. Vet. Zootec. 61(1):93-102.

Matope G., Bhebhe E., Muma J.B., Oloya J., Madekurozwa R.L., Lund A. \& Skjerve E. 2011. Seroprevalence of brucellosis and its associated risk factors in cattle from smallholder dairy farms in Zimbabwe. Trop. Anim. Health Prod. 43(5):975-982.

Microsoft Corporation 2007. Microsoft Access, versão 13.0. Microsoft Corporation, Redmont.

Moreno E., Cloeckaert A. \& Moriyón I. 2002. Brucella evolution and taxonomy. Vet. Microbiol. 90:209-227.

Murray N. 2004. Handbook on Import Risk Analysis for Animals and Animal Products. Vol.2. Quantitative Risk Assessment. OIE, Paris. 126p.

Nunes L.N. 2007. Métodos de imputação de dados aplicados à área de saúde. Tese de Doutorado, Instituto de Matemática. Universidade Federal do Rio Grande do Sul, Porto Alegre, RS. 120p.

OIE 2009. Manuals of Diagnostic Tests and Vaccines for Terrestrial Animals. Chapter 2.4.3 (Bovine brucellosis). Disponível em <http://www. oie.int/fileadmin/Home/eng/Health_standards/tahm/2.04.03_BOVINE_BRUCELL .pdf $>$. Acesso em 22 out. 2014.

Pappas G., Papadimitriou P., Akritidis N., Christou L. \& Tsianos E.V. 2006. The new global map of human brucellosis. Lancet, Infect. Dis. 6(2):91-99.

Perez A.M., Ward M.P., Torres P. \& Ritacco V. 2002. Use of spatial statistics and monitoring data to identify clustering of bovine tuberculosis in Argentina. Prev. Vet. Med. 56(1):63-74.

Perrin J.B., Ducrot C., Vinard J.L., Morignat E., Calavas D. \& Hendrikx P. 2012. Assessment of the utility of routinely collected cattle census and disposal data for syndromic surveillance. Prev. Vet. Med. 105(3):244-252.

Poester F., Figueiredo V.C.F., Lôbo J.R., Gonçalves V.S.P., Lage A.P., Roxo E., Mota P.M.P.C., Müller E.E. \& Ferreira Neto J.S. 2009. Estudos de prevalência da brucelose bovina no âmbito do Programa Nacional de Controle e Erradicação de Brucelose e Tuberculose: Introdução. Arq. Bras. Med. Vet. Zootec. 61(1):1-5.

QGIS Development Team 2014. QGIS Geographic Information System. Open Source Geospatial Foundation Project.

Radostits O.M., Arundel J.H., Gay C.C., Blood D.C. \& Hinchcliff K.W. 2007. Veterinary Medicine: A textbook of the diseases of cattle, horses, sheep, pigs and goats. 10th ed. Saunders Elsevier, Philadelphia. 2065p.

Rushton J. 2009. The economics of animal health and production. CABI, Oxfordshire. 29p.

Salman M.D. 2003. Surveillance and monitoring systems for animal health programs and disease surveys. In: Salman M.D (Ed.), Animal Disease Surveillance and Survey Systems: methods and applications. Blackwell Publishing, Iowa. 222p.

Schaefer J.L. 1999. Multiple Imputation: a primer. Stat. Methods Med. Res. 8:3-15.

SEAPI 2008. Relatórios Epidemiológicos Mensais do ano 2008.

Seleem M.N., Boyle S.M. \& Sriranganathan N. 2010. Brucellosis: a reemerging zoonosis. Vet. Microbiol. 140:392-398.

Seo S. 2006. A review and comparison of methods for detecting outliers in univariate data sets. Dissertação de Mestrado, School of Public Health, University of Pittsburgh, Pittsburgh, PA. 59p.

Skuce R.A., Allen A.R. \& McDowell S.W.J. 2012. Herd-level risk factors for bovine tuberculosis: a literature review. Vet. Med. Int., p.1-10.

Thrusfield M. 2007. Veterinary Epidemiology. Blackwell Science, Oxford. $626 \mathrm{p}$.

Todeschini B. 2010. Enfoque epidemiológico em dados de tuberculose e brucelose visando a implantação de um sistema informatizado de monitoramento e vigilância. Dissertação de Mestrado, Faculdade de Veterinária, Universidade Federal do Rio Grande do Sul, Porto Alegre, RS. 94p.

Todeschini B., Heineck A., Almeida J., Groff A.C.M., Machado G. \& Corbellini L.G. 2012. Social network analysis on innovative governance arrangements for the control of bovine brucellosis and tuberculosis in Brazil. Book of abstracts of the 13th International Symposium on Veterinary Epidemiology and Economics (13th ISVEE): 323

Wells J.S., Ebel E.D., Williams M.S., Scott A.E., Wagner B.A. \& Marshall K.L. 2009. Use of epidemiologic information in targeted surveillance for population inference. Prev. Vet. Med. 89(1/2):43-50.

WHO 2009. Seven Neglected Endemic Zoonoses: some basic facts. Disponível em <http://www.who.int/zoonoses/ neglected zoonotic diseases/en/> Acesso em 24 out. 2010. 